\title{
A FUNCTIONAL CALCULUS FOR GENERAL OPERATORS IN HILBERT SPACE
}

\author{
BY \\ MORRIS SCHREIBER( $(1)$
}

1. Introduction. The force of the spectral theorem for normal operators in Hilbert space is that it affords a reduction of operator-theoretic problems to function theoretic ones. Specifically, if $A$ is a normal operator $\left(A A^{*}=A^{*} A\right)$ then there is an isometric isomorphism between the (weakly closed) algebra $\mathfrak{A}$ of operators generated by $A, A^{*}$ and $I$ (the identity operator) and a (normed) algebra $\mathfrak{F}$ of numerical functions. Thus to each function $f \in \mathfrak{F}$ there corresponds an operator $f(A) \in \mathfrak{A}$, and the mapping $f \rightarrow f(A)$ preserves all algebraic operations as well as norms. This mapping is appropriately called a functional calculus for the operator $A$. If $A$ is a general (not necessarily normal) operator, so that the spectral theorem does not apply, it is nevertheless possible to develop a functional calculus for $A$, in the sense that one can find an algebra $\mathfrak{A}$ properly containing the polynomials in $A$, a ring of functions $\mathfrak{F}$, and an algebra homomorphism of $\mathfrak{F}$ onto $\mathfrak{A}$ which is continuous in some sense. Following a suggestion which apparently goes back to Cartan, Cauchy's integral formula has been used to establish such a representation for general operators [9], with positive though limited results. The main limitation of this method is that it is purely Banach-space-theoretic and so makes no use of the Hilbert space structure. The Gelfand-Neumark theory [2] on the representation of Banach algebras with involution, another possible approach to the problem, is also ineffective because the powerful results of the theory demand both commutativity and the presence of an involution, and algebras generated by non-normal operators do not have both of these properties. A third approach, which we shall describe, consists in generalizing the measuretheoretic approach to spectral theory for normal operators, and leads to some new results. The generalization consists in considering operator valued "measures," termed operator measures, which are related to general operators as spectral measures are to normal operators, and which differ from spectral measures in that they are positive-operator-valued rather than projectionvalued (a projection is positive and idempotent, so that technically the generalization is simply the relaxation of the condition of idempotence). The application of operator measures to the functional representation of general operators proceeds from the fact (essentially proved by Nagy [6]) that every

Received by the editors August 20, 1956.

1 The author wishes to thank Professor P. R. Halmos for his guidance in this work, which is essentially part of a doctoral dissertation submitted to the University of Chicago. 
operator $A$ (subject to the innocuous technical restriction that $\|A\| \leqq 1$ ) determines a unique operator measure $F$, supported on the unit circle in the complex plane, and such that $A^{n}=\int z^{n} d F(z), n=0,1,2, \cdots$, where the integral is the usual type of spectral integral (weak or strong topology), and we pursue the possibility of forming functions of $A$ by the formula $f(A)$ $=\int f(z) d F(z)$ with the object of representing $A$ by functions on the unit circle. Our main results are that for operators $A$ whose operator measure is equivalent to (has the same null sets as) Lebesgue measure the mapping $f \rightarrow f(A)$ is a norm-decreasing algebra homomorphism from the algebra of bounded boundary values of functions analytic inside the circle (with supremum norm) into the strong closure of the algebra $\mathfrak{A}$ generated by $A$ and $I$; and if moreover the spectrum of $A$ contains the unit circle, then the above mapping is an isometry of the subalgebra of continuous boundary values onto the uniform closure of $\mathfrak{A}$. These results are incomplete on two points: (i) we lack a characterization of operators with operator measure equivalent to Lebesgue measure and spectrum containing $C$, and (ii) we have no information on the class of representing functions associated to operators whose operator measure is not equivalent to Lebesgue measure.

We conclude the paper with a theorem showing the relation between the spectral measure and the operator measure of a normal contraction, by means of which normality can be characterized in terms of operator measures.

2. We are concerned with complex Hilbert space, and we use the word operator to mean bounded linear transformation. The symbol $C$ is reserved throughout for $\{z:|z|=1\}$ and $m$ is Lebesgue measure on $C$, normalized so that $m(C)=1$. The spectrum of an operator $A$ is denoted $\operatorname{Sp}(A)$ and we write $\Lambda(F)$ for the support (complement of the union of all open sets where $F$ vanishes) of a countably additive function $F$ of Borel sets.

We shall make use of the basic definitions and facts about operator measures and unitary dilations, as expounded in [10]. We state them here briefly for the convenience of the reader.

An operator measure is a function $F$ from Borel sets on the unit circle $C$ to positive operators on a Hilbert space $H$ which is weakly (and therefore strongly) countably additive, and is normalized so that $F(C)=I$ (the identity operator). If $f$ is a complex-valued function bounded and Borel measurable on the support $\Lambda(F)$ of $F$ then the integral $\int f(z) d F(z)$ may be understood in either the weak or strong topology and defines an operator $T$ such that $(T x, y)=\int f(z) d(F(z) x, y)=\left(\left[\int f(z) d F(z)\right] x, y\right)$ for all $x$ and $y$ in $H$. An operator measure $F$ on $H$ determines, uniquely within unitary equivalence, a larger space $K \supset H$ and a spectral measure $E$ (supported in $C$ ) on $K$ such that if $P$ is the projection of $K$ onto $H$ then $F(\sigma) x=P E(\sigma) x$ for every Borel subset $\sigma \subset C$ and every $x \in H$ (we may express this intrinsically by writing $F(\sigma)$ $=P E(\sigma) P$, where $F$ is now regarded as operating on all of $K$ and mapping $K \ominus H$ to 0$)$; moreover, no subspace of $K \ominus H$ reduces all the projections 
$E(\sigma) \neq 0[8]$. The space $K$ and spectral measure $E$ are called respectively the minimal dilation space and minimal dilation of $F$, and the unitary operator $U$ on $K$ defined by $E$ (that is, $U=\int z d E(z)$ ) is called the minimal dilation of the operator $f z d F(z)$. An operator measure $F$ is called strong if $\int z^{n} d F(z)=\left[\int z d F(z)\right]^{n}$ for all $n$. Every contraction operator $A$ (that is, $\|A\| \leqq 1$ ) determines a unique strong operator measure $F$, and every strong operator measure arises in this way.

3. To simplify the exposition we shall consider only contraction operators. Since every operator is a scalar multiple of a contraction, it will be apparent that the results are valid, with minor and obvious modifications, for all operators.

Lemma 1. If $F$ is an operator measure on $H$ and $E$ is its minimal dilatation on $K \supset H$, then, for every Borel set $\sigma$, we have $E(\sigma)=0$ if and only if $F(\sigma)=0$.

Proof. By hypothesis $F(\sigma)=P E(\sigma) P$, where $P$ is the projection on $H$. If $E(\sigma)=0$ then $F(\sigma)=0$ trivially. Conversely if $F(\sigma)=P E(\sigma) P=0$, then $0=P[E(\sigma)]^{2} P=[P E(\sigma)][P E(\sigma)]^{*}$, and consequently $P E(\sigma)=E(\sigma) P=0$. This means that the range $M$ of $E(\sigma)$ is a subspace of $K \ominus H$. For every Borel set $\delta, E(\delta) E(\sigma)=E(\sigma) E(\delta)$; that is, $M$ reduces $E$. This contradicts the minimality of $E$ unless $E(\sigma)=0$, as was to be shown.

Corollary 1. $\Lambda(F)=\Lambda(E)$, where $E$ and $F$ are as in Lemma 1.

Observe that, with $E$ and $F$ as in Lemma 1,

$$
(E(\sigma) x, x)=(F(\sigma) x, x)
$$

for all Borel sets $\sigma$ and all $x$ in $H$, because $(F(\sigma) x, x)=(P E(\sigma) P x, x)$ $=(E(\sigma) P x, P x)=(E(\sigma) x, x)$. This is a weaker assertion than that of Lemma 1.

If $G$ is either an operator measure or a spectral measure we define the essential supremum, denoted $\|f\|_{\infty}$, of a function $f$ on $\Lambda(G)$ exactly as in the case of numerical measures.

$$
\|f\|_{\infty}=\inf _{G(\delta)=I} \sup _{z \in \delta}|f(z)| .
$$

The set of all Borel measurable functions on $\Lambda(G)$ with finite essential supremum is a normed ring, with essential supremum as norm, if we identify functions agreeing except on $G$-null sets. We denote this normed ring by $L_{\infty}(G)$.

CoRollary 2. If $F$ is an operator measure and $E$ is its minimal dilation then $L_{\infty}(E)=L_{\infty}(F)$.

This is immediate from Lemma 1.

Definition 1. Let $A$ be a contraction with strong operator measure $F$. For any function $f \in L_{\infty}(F)$ we define an operator $f(A)$ by the integral $\int f(z) d F(z)$, which may be interpreted in either the weak or strong topology. Thus, 


$$
(f(A) x, y)=\left(\left[\int f(z) d F(z)\right] x, y\right)=\int f(z) d(F(z) x, y)
$$

for every $x$ and $y$ in $H$. We denote the mapping $f \rightarrow f(A)$ by $\phi$.

Lemma 2. Let $A$ be a contraction with strong operator measure $F$ on $H$ and let $U=\int z d E(z)$ be its dilation on the minimal dilation space $K$. Then $f(A)$ $=P f(U) P$, where $P$ is the projection of $K$ onto $H$.

Proof. By the spectral theorem $f(U)$ is well defined for any $f$ in $L_{\infty}(E)$ $=L_{\infty}(F)$. If $x$ and $y$ are in $H$ then $(f(A) x, y)=\int f(z) d(F(z) x, y)=\int f(z) d(E(z) x, y)$ by (1), and the last integral is equal to $(f(U) x, y)=(P f(U) P x, y)$. Since this holds for all $x$ and $y$ in $H$, the proof is complete.

Let $\Delta(F)$ denote the set of all complex valued functions which are approximable on $\Lambda(F)$ pointwise almost everywhere with respect to $F$ by uniformly bounded sequences of polynomials in $z$. We shall refer to this notion of convergence as bounded pointwise convergence and abbreviate it (BP).

LEMma 3. $\Delta(F) \subset L_{\infty}(F)$, and convergence $(B P)$ within $\Delta(F)$ implies strong convergence within $\phi\left[L_{\infty}(F)\right]$

Proof. The inclusion is clear. To prove the second assertion we must show that if $\left[f_{n}\right]$ is a sequence of functions in $\Delta(F)$ converging (BP) to $f$ then the sequence $\left[f_{n}(A)\right]$ of operators converges strongly to $f(A)$. For any $x$ in $H$ Lemma 2 implies that

$$
\left\|\left[f_{n}(A)-f(A)\right] x\right\|^{2}=\left\|\left[P\left(f_{n}(U)-f(U)\right) P\right] x\right\|^{2}
$$

which does not exceed

$$
\left\|\left[f_{n}(U)-f(U)\right] x\right\|^{2}=\int\left|f_{n}(z)-f(z)\right|^{2} d(E(z) x, x) .
$$

By (1) and the assumption on $\left[f_{n}\right]$ the integrand is bounded and converges to 0 pointwise almost everywhere with respect to the measure $d(E(z) x, x)$. Therefore by the Lebesgue bounded convergence theorem the integral, and hence also the quantity $\left\|\left[f_{n}(A)-f(A)\right] x\right\|$, converges to 0 as $n$ tends to $\infty$. Since this holds for all $x$ in $I$, the proof is complete.

THEOREM 1. The map $\phi$ is a norm decreasing * vector space homomorphism of $L_{\infty}(F)$, and restricted to $\Delta(F)$ it also preserves products. ( $A *$ mapping sends complex conjugation into adjunction.)

Proof. In the notation of Lemma 2, if $f$ is in $L_{\infty}(F)$ then $\|\phi f\|=\|f(A)\|$ $=\|P f(U) P\| \leqq\|f(U)\|$, which is equal to $\|f\|_{\infty}$ by the spectral theorem( $\left.{ }^{2}\right)$,

(2) That ||$f(A)|| \leqq|| f||_{\infty}$ for $f$ analytic in a domain containing $\mathrm{Sp}(A)$ was proved by v. Neumann [7] and later by Heinz [3] and Nagy [6]. The above argument applies Nagy's method to functions in $L_{\infty}(F)$. 
and $\phi \bar{f}=\bar{f}(A)=P \bar{f}(U) P=P\left[f(U)^{*}\right] P=[P f(U) P]^{*}=[\phi f]^{*}$. That $\phi$ is a vector space homomorphism is clear (it is not in general an isomorphism; for example, if $A^{2}=0$ then $\phi\left[z^{2}\right]=0$ but $z^{2} \neq 0$ everywhere on $\left.C\right)$. The last statement of the theorem is the assertion that $f g(A)=f(A) g(A)$ for $f$ and $g$ in $\Delta(F)$. We observe that because $F$ is a strong operator measure we have $\int z^{n} d F(z) \int z^{m} d F(z)$ $=\left[\int z d F(z)\right]^{n}\left[\int z d F(z)\right]^{m}=\left[\int z d F(z)\right]^{n+m}=\int z^{n+m} d F(z)$ for all non-negative integers $n, m$, and therefore, if $p$ and $q$ are polynomials in $z$ then $p(A) q(A)$ $=\int p(z) d F(z) \int q(z) d F(z)=\int p(z) q(z) d F(z)=p q(A)$. This is the essential use of the strong property of $F$. Let $\left[p_{n}\right]$ and $\left[q_{n}\right]$ be sequences of polynomials in $z$ converging (BP) to $f$ and $g$ respectively. By Lemma $3, p_{n}(A)$ and $q_{n}(A)$ converge strongly to $f(A)$ and $g(A)$ respectively. The sequences $\left[p_{n}(A)\right]$ and $\left[q_{n}(A)\right]$ are uniformly bounded in norm because they are strongly convergent (or one may use the first assertion of the present theorem) and their limits have the same bound. Multiplication being jointly continuous in the strong topology on bounded sets it follows that $p_{n}(A) q_{n}(A)$ converges strongly to to $f(A) g(A)$. From the fact that $\left[p_{n}\right]$ and $\left[q_{n}\right]$ converge (BP) to $f$ and $g$ respectively it follows that $\left[p_{n} q_{n}\right]$ converges $(\mathrm{BP})$ to $f g$, and hence that $\left(p_{n} q_{n}\right)(A)$ converges strongly to $(f g)(A)$. But $\left(p_{n} g_{n}\right)(A)=p_{n}(A) q_{n}(A)$ for all $n$. Hence $(f g)(A)=\lim \left(p_{n} q_{n}\right)(A)=\lim p_{n}(A) q_{n}(A)=f(A) g(A)$ and the proof is complete.

TheOREM 2. Let $A$ be a contraction with strong operator measure $F$. If $A$ is not unitary then $\Lambda(F)=C$.

Proof. Suppose to the contrary that $\Lambda(F) \neq C$, so that $\Lambda(F)$ does not separate the plane. Then every continuous function on $\Lambda(F)$ is a limit uniformly on $\Lambda(F)$ of polynomials in $z$ (see [5]). In particular $f(z)=\bar{z}$ is such and so belongs to $\Delta(F)$. Then by the preceding theorem $I=\phi(1)=\phi(z \bar{z})$ $=\phi(z) \phi(\bar{z})=A A^{*}$. Similarly $I=A^{*} A$ and the theorem is proved.

Thus the functions in $\Delta(F)$ are limits (BP) everywhere on $C$, and $\phi$ is a continuous $*$ algebra homomorphism of $\Delta(F)$ into the ring of operators generated by $A$ and $I$. We specialize now to the class $\&$ of contractions whose operator measures are equivalent to Lebesgue measure $m$ on $C$. That is, $A=\int z d F(z)$ belongs to $\mathfrak{R}$ if $F$ has exactly the same null sets as $m$. We have shown in [10] that all operators $A$ such that $\|A\|<1$ belong to $\&$, as do all quasi-nilpotent operators.

THEOREM 3. If $A=\int z d F(z)$ belongs to $\&$ then $\Delta(F)$ is exactly the set of $f$ in $L_{\infty}(F)$ such that $\int z^{n} f(z) d m(z)=0$ for $n>0$.

Proof. Under the present assumption limits almost everywhere with respect to $F$ or $m$ are the same. Let $f$ be in $\Delta(F)$, with $f=\lim p_{n}(\mathrm{BP})$. If $k>0$ then $\int p_{n}(z) z^{k} d m(z)=0$. Hence $\left|\int z^{k} f(z) d m(z)\right|=\left|\int\left(f(z)-p_{n}(z)\right) z^{k} d m(z)\right|$ $\leqq \int\left|f(z)-p_{n}(z)\right| d m(z)$ for $k>0$. The last integral converges to 0 by the Lebesgue bounded convergence theorem, showing that $f$ has the property stated in the theorem. Conversely, if $f$ has the stated property and is in $L_{\infty}(F)$, 
then its Fourier coefficients $c_{n}=\int \bar{z}^{n} f(z) d m(z)$ vanish for $n<0$. It follows that the Fejer means $\sigma_{n}(z)=(1 / n) \sum_{n}^{n} c_{k} z^{k}$ of $f$ are polynomials in $z$ alone. Moreover the $\sigma_{n}$ are uniformly bounded by the same bound as $f[12,3.22]$. Since the measure of $C$ is finite $f$ is in $L_{1}(m)$. Then by the Fejer-Lebesgue theorem $[11$, p. 415$]$ the sequence of Fejer means converges to $f$ almost everywhere, hence (BP), so that $f$ is in $\Delta(F)$ and the proof is complete.

Corollary 3. If $A=\int z d F(z)$ is in $\mathbb{R}$ then $\Delta(F)$ is closed under limits $(B P)$ and consists of all nontangential boundary values, almost everywhere on $C$, of functions analytic and bounded in the interior of $C$.

Proof. That $\Delta(F)$ is closed under limits (BP) follows at once from the integral formula of Theorem 3 . To prove the second assertion, observe that if $f$ is in $\Delta(F)$ then the Fejer means $\sigma_{n}$ of $f$ are analytic in the interior of $C$ (since they are polynomials) and are uniformly bounded there (by the maximum modulus theorem). Hence, by a consequence of Vitali's theorem [11, p. 168] on the uniform convergence of bounded sequences of analytic functions, a subsequence $\sigma_{n_{i}}$ converges uniformly on every closed subset of the interior of $C$ and so defines a bounded analytic function in the interior of $C$. It is known that such a function assumes a bounded boundary value on $C$ almost everywhere, nontangentially $[12, \mathrm{pp} .86,44]$, and it is easy to check that this boundary value coincides with the given function $f$.

Thus for operators in $\&$ the map $\phi$ is an algebra homomorphism on the boundary values of bounded analytic functions. It is clear from Lemma 3 that $\phi[\Delta(F)]$ is always contained in the strong sequential closure of the algebra $\mathfrak{A}$ generated by $A$ and $I$, for any contraction $A$. To say more about the range of $\phi$ we specialize still further. Write $\|f\|_{S}$ for the spectral norm $\sup [|f(z)|: z$ in $\operatorname{Sp}(A)]$ of a function $f$.

Lemma 4. If $f$ in $L_{\infty}\left(F_{A}\right)$ is analytic in a domain containing $\operatorname{Sp}(A)$ then $\|f\|_{s} \leqq\|f(A)\| \leqq\|f\|_{\infty}$.

Proof. By the Dunford spectral mapping theorem [4, p. 122] $\|f\|_{S}$ is the spectral radius of $f(A)$ and therefore is not greater than $\|f(A)\|$. The second inequality is the first assertion of Theorem 1.

Theorem 4. If $A$ is in $\mathbb{R}$ and $\mathrm{Sp}(A) \supset C$ then $\phi$ is an isometry of the space of continuous functions in $\Delta(F)$ onto the uniform closure $\overline{\mathfrak{A}}$ of $\mathfrak{A}$, and so in particular $\overline{\mathfrak{A}} \subset \phi[\Delta(F)]$.

Proof. Let $B$ be an arbitrary element of $\overline{\mathfrak{A}}$. Then $B$ is the uniform limit of a sequence $p_{n}(A)$ of polynomials in $A$. If $p$ is a polynomial in $z$ then $\|p\|_{S}$ $\leqq\|p(A)\| \leqq\|p\|_{\infty}$ by the preceding lemma, and since $\operatorname{Sp}(A) \supset C$ we have $\|p\|_{S}=\|p\|_{\infty}$ by the maximum modulus theorem. Hence $\left\|p_{n}\right\|_{\infty}=\left\|p_{n}(A)\right\|$ for all $n$. Consequently the polynomials $p_{n}$ converge uniformly on $\operatorname{Sp}(A)$, and in particular on $C$, to a continuous function $f$ in $\Delta(F)$. Hence $p_{n}(A)$ converges 
uniformly to $f(A)$, so that $B=f(A)$. This shows that every element of $\overline{\mathfrak{A}}$ has the form $f(A)$ for $f$ continuous in $\Delta(F)$. In the other direction, if $g$ is continuous in $\Delta(F)$ with $A$ in $\mathbb{R}$, then, just as in Theorem 3 , the Fejer means $\sigma_{n}$ are polynomials in $z$, and by a corollary of the Fejer theorem [11, p. 414] $\sigma_{n}$ converges to $f$ uniformly on $C$. Hence $g(A)$ is the uniform limit of $\sigma_{n}(A)$ and so belongs to $\overline{\mathfrak{A}}$. This also shows that the polynomials in $z$ are uniformly dense in the continuous functions in $\Delta\left(F_{A}\right)$, and since $\phi$ is isometric on the polynomials it is isometric throughout. This completes the proof.

Note that the set of continuous functions in $\Delta\left(F_{A}\right)$, with $A$ in \&, is exactly the set of continuous boundary values of functions analytic inside $C$. Thus we have a true representation for contractions $A$ in $\&$ such that $\operatorname{Sp}(A) \supset C$.

A more illuminating description of operators of this type is not at hand. One example is the so-called unilateral shift operator $U$, which is defined on the span of the functions $z^{n}, n=0,1,2, \cdots,|z|=1$, by the relation $U f(z)$ $=z f(z)$. The unitary dilation $\mathfrak{U}$ of $U$ is defined on the space $L_{2}(m)$, that is, the span of all powers, both positive and negative, of $z$, by the relation $\mathfrak{u} f(z)=z f(z)$. (This may be verified by direct calculation from the definitions, or may be found in [10].) The spectral measure $E$ of $\mathfrak{U}$ is given by the relation $E(\delta) f(z)=k_{\delta}(z) f(z)$, where $k_{\delta}$ is the characteristic function of the Borel set $\delta$, so that $E$ is equivalent to $m$. But $E$ is the minimal dilation of the operator measure $F$ of $U$, whence it follows by Lemma 1 that $F$ is also equivalent to $m$. Moreover we have $\operatorname{Sp}(U)=\{z:|z| \leqq 1\} \supset C$ (if $|\alpha|<1$ then $U^{*}\left(\sum_{0} \alpha^{n} z^{n}\right)$ $=\sum_{0} \alpha^{n+1} z^{n}=\alpha\left(\sum_{0} \alpha^{n} z^{n}\right)$, so that $\alpha \in \operatorname{Sp}\left(U^{*}\right)$, and it follows that $\operatorname{Sp}(U)$ $=\operatorname{Sp}\left(U^{*}\right)$ is the whole disc). Thus $U$ has the stated properties.

In the classical spectral theory of a general operator $A$ one considers functions $f$ analytic in a domain $D_{f}$, depending on $f$, properly containing $\mathrm{Sp}(A)$, and one writes

$$
f(A)=\frac{1}{2 \pi i} \int_{\gamma} f(z) R(z) d z
$$

where $R(z)=(I-z A)^{-1}$ is the resolvent of $A$, and $\gamma$ is a contour within $D_{f}$ and containing $\operatorname{Sp}(A)$ in its interior. This defines an algebra homomorphism $\psi$ from the set of all such functions to operators, such that $\psi(1)=I$ and $\psi(z)$ $=A[9$, Chap. XI $]$. J. von Neumann has recently shown that $\psi$ is also norm decreasing if the functions are given their spectral norm (see footnote to Theorem 1 above). These results are improved somewhat by the present method in two senses. First, if $\operatorname{Sp}(A) \supset C$ then we may include functions which need not be analytic in a domain properly containing $\operatorname{Sp}(A)$; and second, the operators $f(A)$ defined by (3) will be contained in the uniform closure of the algebra generated by $A$ and $I$, and this is a smaller algebra than the strong sequential closure.

The two methods are coherent. That is, if $f$ is analytic in a domain containing the unit disc then 


$$
\int_{C} f(u) d F(u)=\frac{1}{2 \pi i} \int_{\gamma} f(z) R(z) d z
$$

where $F$ is the strong operator measure of a contraction $A, R(z)$ is its resolvent, and $\gamma$ is a contour containing $C$ in its interior. For the power series expansion of $f$ at the origin will converge in a circle larger than $C$, so that its partial sums $s_{n}$ will converge uniformly on $C$. Hence $\int s_{n}(u) d F(u)$ will converge uniformly to $\int f(u) d F(u)$. But $\int s_{n}(u) d F(u)=s_{n}(A)$, and it is easy to see, just as in the numerical case, that $s_{n}(A)$ converges uniformly to $(1 / 2 \pi i)$ $\cdot \int_{\gamma} f(z) R(z) d z$.

If $A$ is a normal operator with spectral measure $E$, and $B$ is any operator, then Fuglede's theorem asserts that $B$ commutes with $A$ if and only if $B$ commutes with all the projections $E(\sigma)$, or equivalently, $B$ commutes with all bounded measurable functions of $A$ [1]. The analogous assertion for an arbitrary operator $A$ with operator measure $F$, namely that $B$ commutes with $A$ if and only if $B$ commutes with all the operators $F(\sigma)$, and hence with all the operators $f(A)$ where $f \in L_{\infty}(F)$, is false. For the function $f(z)=\bar{z}$ belongs to $L_{\infty}(F)$, so that the assertion would entail as a consequence the further assertion that $B$ commutes with $A$ if and only if $B$ commutes with $A^{*}$, and this is easily refuted by matricial examples. The only possible theorem is the following.

THEOREM 5. Let $A=\int z d F(z)$ be an arbitrary operator with strong operator measure $F$, and let $B$ be an operator. Then $B$ commutes with $A$ and $A^{*}$ if and only if $B$ commutes with all $f(A)$ with $f$ in $L_{\infty}(F)$.

Proof. Let $A^{(n)}$ denote $A^{n}$ if $n \geqq 0$ and $A^{*|n|}$ if $n<0$. Then clearly $B$ commutes with $A$ and $A^{*}$ if and only if $B$ commutes with $A^{(n)}$ for all $n$, which is so if and only if $\left(A^{(n)} B x, y\right)=\left(B A^{(n)} x, y\right)=\left(A^{(n)} x, B^{*} y\right)$ for all $x$ and $y$ in $H$ and all $n$. We use $F$ to express these quantities as follows.

$$
\begin{aligned}
& \left(A^{(n)} B x, y\right)=\int z^{n} d(F(z) B x, y), \\
& \left(B A^{(n)} x, y\right)=\int z^{n} d\left(F(z) x, B^{*} y\right) \int z^{n} d(B F(z) x, y) .
\end{aligned}
$$

Fixing $x$ and $y$ for the moment, the set functions $(F(\cdot) B x, y)$ and $(B F(\cdot) x ; y)$ are both complex valued measures on the circle $C$, and the left hand members of (4) and (5) are the values of the functionals induced by these measures on the functions $f(z)=z^{n}, n=0, \pm 1, \pm 2, \cdots$. But these functions span the ring of continuous functions on $C$. Hence $\left(A^{(n)} B x, y\right)=\left(B A^{(n)} x, y\right)$ for all $n$ if and only if the two measures induce the same functional and hence are equal as measures; that is, if and only if $(F(\sigma) B x, y)=(B F(\sigma) x, y)$ for all Borel sets $\sigma$ in $C$. The argument does not depend on the choice of $x$ and $y$, so that we may now let them be arbitrary, and this completes the proof. 
Corollary 4. If $A$ commutes with all $F(\sigma)$ then $A$ is normal and conversely.

Proof. We merely replace $B$ by $A$ in the above argument.

4. If $A$ is a normal contraction then it determines both a spectral measure $E$ supported on $\operatorname{Sp}(A)$ and a strong operator measure $F$ supported on $C$. We propose to express $F$ in terms of $E$. The first step is to consider operators of the form $A_{a}=a I$, where $a$ is a complex number. Then $\operatorname{Sp}\left(A_{a}\right)=\{a\}$ and the spectral measure $E_{a}$ of $A_{a}$ assigns $I$ to the set $\{a\}$. If $|a|<1$ then $A_{a}$ is a proper contraction in the terminology of [10] and we may apply the results of that paper. In particular, it is shown there that the strong operator measure $F$ of a proper contraction $A$ is given by

$$
F(\sigma)=\int_{\sigma} K(A, z) d m(z)
$$

where $K(A, z)=\sum_{-\infty}^{\infty} \bar{z}^{n} A^{(n)}$ is the so-called operator function of $A$. In the present simple case $K(A, z)$ reduces to $K_{a}(z) \cdot I$, where $K_{a}(z)$ is the Poisson kernel $[12$, p. 51$]$, and the formula (6) is easily checked by direct calculation. Thus, denoting by $F_{a}$ the strong operator measure of $A_{a}$, we may write $F_{a}(\delta)$ $=\int_{\delta} K_{a}(z) \operatorname{Idm}(z)$. Since for $|a|<1$ the function $K_{a}$ is positive on $C$ we may define a family of measures $m_{a}$ on $C$ as follows.

$$
m_{a}(\delta)=\int_{\delta} K_{a}(z) d m(z), \quad|a|<1 .
$$

The behavior of $m_{a}$ as $a$ approaches the boundary is described by

Lemma 5. If $f$ is continuous at $u \in C$ then $\lim _{r=1} \int f(z) d m_{r u}(z)=f(u)$.

Proof. The one parameter family of functions

$$
\left\{K_{r u}: 0 \leqq r<1,|u|=1\right\}
$$

constitutes a positive kernel for each fixed $u \in C$, in the sense of $[12$, pp. 45 , 51]. The lemma then follows as an immediate application of $[12,3.21]$ to this kernel.

This being so, we may extend the definition of $m_{a}$ to the boundary by taking $m_{a}$ to be the measure concentrated at the point $z=a$ when $|a|=1$. With $m_{a}$ so extended we may now write

$$
F_{a}(\delta)=m_{a}(\delta) I,
$$$$
|a| \leqq 1 \text {. }
$$

Now let $A$ be a normal contraction with spectral measure $E$. It follows that $\operatorname{Sp}(A)=\Lambda(E)$ is contained in the unit disc $D$. Heuristically speaking, every point $z$ of $\operatorname{Sp}(A)$ contributes the amount $m_{z}(\delta) I$ to the value of $F(\delta)$, where $F$ is the strong operator measure of $A$. By adding these contributions we are led to 
THEOREM 6. If $A$ is a normal contraction with spectral measure $E$ then its strong operator measure $F$ is given by

$$
F(\delta)=\int_{D} m_{z}(\delta) d E(z)
$$

Proof. For any fixed Borel set $\delta$ the function $m_{z}(\delta)$ defined by (7), and extended as noted, is bounded, measurable and positive on all of $D$, so that the set function $F$ given by (9) is positive-operator-valued. Let $\left\{\delta_{i}\right\}$ be a countable family of disjoint Borel sets, and write $f_{n}(z)=\sum_{1}^{n} m_{z}\left(\delta_{i}\right)$. Then $1 \geqq f_{n}(z) \geqq 0$ for all $n$ and all $z \in D$, and $f_{n}(z)$ converges pointwise everywhere in $D$ to $m_{z}\left(\cup_{i=1}^{\infty} \delta_{i}\right)$ because $m_{z}$ is for each $z$ a measure. For any vector $x$ in $H$, $\left(F\left(\bigcup_{i=1}^{n} \delta_{i}\right) x, x\right)=\int_{D} m_{z}\left(\bigcup_{i=1}^{n} \delta_{i}\right) d(E(z) x, x)=\int_{D} f_{n}(z) d(E(z) x, x)$. By the Lebesgue bounded convergence theorem the last integral converges to $\int_{D} m_{z}\left(\cup_{i=1}^{\infty} \delta_{i}\right) d(E(z) x, x)=\left(F\left(\bigcup_{i=1}^{\infty} \delta_{i}\right) x, x\right)$ as $n$ tends to $\infty$. That is, $F$ is weakly countably additive. It follows that $F$ is strongly countably additive [10]. Moreover $F(C)=\int_{D} m_{z}(C) d E(z)=\int_{D} 1 d E(z)=I$, so that $F$ is an operator measure. It remains only to show that $\int_{C} z^{k} d F(z)=A^{(k)}$ for $k \neq \neq 0$. Let $\left\{\delta_{i}\right\}$ be a disjoint partition of $C$ into Borel sets and let $z_{i}$ be a point of $\delta_{i}$. Then $\sum_{1}^{n} z_{i}^{k} F\left(\delta_{i}\right)=\sum_{1}^{n} z_{i}^{k} \int_{D} m_{z}\left(\delta_{i}\right) d E(z)=\int_{D}\left\{\sum_{1}^{n} z_{i}^{k} m_{z}\left(\delta_{i}\right)\right\} d E(z)$. The expression in braces is an approximating sum for $\int_{C} u^{k} d m_{z}(u)$, where $m_{z}$ has its extended sense, and this integral has the value $z^{k}$ for every $z$ in the closed unit disc. Thus, as the partition is refined indefinitely $\sum_{1}^{n} z_{i}^{k} m_{z}\left(\delta_{i}\right)$ converges to $z^{k}$ so that $\sum_{1}^{n} z_{i}^{k} F\left(\delta_{i}\right)$ converges to $\int_{\mathrm{Sp}(A)} d E(z)=A^{k}$. Since this holds for all $k>0$, and therefore by adjunction for all $k<0$, the proof is complete.

Corollary 5.1. A contraction $A$ is normal if and only if the set of positive operators determined by its strong operator measure is a commutative set.

Proof. If $A$ is normal then for all Borel sets $\alpha$ and $\beta$ we have $F(\alpha) F(\beta)$ $=F(\beta) F(\alpha)=\int_{D} m_{z}(\alpha) m_{z}(\beta) d E(z)$, where $F$ is the strong operator measure of $A$, by Theorem 6 and the spectral theorem. If, conversely, $\{F(\cdot)\}$ is commutative, then for any fixed Borel set $\alpha$ the operator $F(\alpha)$ commutes with all the $F(\beta)$, which easily implies that $F(\alpha)$ commutes with $\int_{C} z d F(z)=A$. Since $\alpha$ may be chosen arbitrarily, we see that $A$ commutes with all the positive operators in the range of $F$, whence it follows by Corollary 4 that $A$ is normal.

Thus normal contractions are distinguished from all others by the commutativity properties of their operator measures (Corollaries 4 and 5.1). As a final corollary we have another analogue of Fuglede's theorem.

CoROllaRY 5.2. If $A$ is a normal contraction with strong operator measure $F$ and $B$ is any operator and $A B=B A$, then $B F(\delta)=F(\delta) B$ for all Borel sets $\delta$.

Proof. Let $E$ be the spectral measure of $A$. Then $B E(\delta)=E(\delta) B$ for all $\delta$ 
by Fuglede's theorem, and therefore $B$ commutes with $\int_{D} m_{z}(\delta) d E(z)=F(\delta)$ for all $\delta$.

\section{BIBLIOGRAPHY}

1. B. Fuglede, A commutativity theorem for normal operators, Proc. Nat. Acad. Sci. U.S.A. vol. 36 (1950) pp. 35-40.

2. I. Gelfand, and M. Neumark, On the imbedding of normed rings into the ring of operators in Hilbert space, Rec. Math. (Mat. Sbornik) N.S. vol. 12(54) (1943) pp. 197-213.

3. E. Heinz, Ein v. Neumannscher Satz über beschrankte Operatoren im Hilbertschen Raum, Nachr. Ges. Wiss. Göttingen (1952) pp. 5-6.

4. E. Hille, Functional analysis and semi-groups, Amer. Math. Soc. Colloquim Publications, vol. 31, New York, 1948.

5. S. N. Mergelyan, On the representation of functions by series of polynomials on closed sets, Amer. Math. Soc. Translations no. 85, 1953.

6. B. v. Sz.-Nagy, Sur les contractions de l'espace de Hilbert, Acta Univ. Szeged. vol. 15 (1953) pp. 87-92.

7. J. v. Neumann, Eine Spektraltheorie fur allgemeine Operatoren eines unitaren Raumes, Math. Nachr. vol. 4 (1951) pp. 258-281.

8. M. A. Neumark, On a representation of additive operator set functions, C.R. (Doklady) Acad. Sci. URSS. vol. 41 (1943) pp. 359-361.

9. F. Riesz, and B. v. Sz.-Nagy, Leçons d'analyse fonctionelle, Academie des Sciences de Hongrie, Akademiai Kiado, Budapest, 1952.

10. M. Schreiber, Unitary dilations of operators, Duke Math. J. vol. 24 (1956) pp. 579-594.

11. E. C. Titchmarsh, Theory of functions, 2d ed., Oxford, 1950.

12. A. Zygmund, Trigonometrical series, Warszawa-Lwow, 1935.

University of Chicago,

Chicago, Ill.

Cornell University,

ITHACA, N. Y. 